\title{
Estabilidad temporal del C.I. y potencial de aprendizaje en niños superdotados: implicaciones diagnósticas
}

\author{
$\mathrm{M}^{\mathrm{a}}$ Dolores Calero* y M $\mathrm{M}^{\mathrm{a}}$ Belén García-Martin
}

Departamento de Personalidad, Evaluación y Tratamiento Psicológico. Universidad de Granada

\begin{abstract}
Resumen: Tradicionalmente la determinación de la sobredotación se ha realizado con tests tradicionales de inteligencia. El principal argumento para hacerlo ha sido la estabilidad temporal de esta medida. En los últimos años algunos autores defienden una determinación temprana de la sobredotación en niños, aunque otros señalan que la determinación del C.I. en niños pequeños arroja un número importante de falsos positivos debido a la variabi lidad de la medida de la inteligencia por influencia de diferentes factores tales como la plasticidad cerebral, la estimulación, etc..., por lo que proponen el uso de índices complementarios para el diagnóstico de sobredotación. En este trabajo se realiza un estudio longitudinal de dos años a 49 niños de entre 5 y 9 años, -inicialmente identificados como superdotados-, para comprobar la estabilidad de su C.I. y de otras medidas tales como el potencial de aprendizaje y la memoria de trabajo. Los resultados muestran como las medidas de potencial de aprendizaje y memoria de trabajo permanecen estables en el tiempo mientras que el C.I. de un grupo de niños de menor edad no se mantiene en dicho periodo. Estos resultados señalan la utilidad de las medidas de P.A. como un índice complementario en la determinación de la sobredotación en niños pequeños.
\end{abstract}

Palabras clave: C.I.; sobredotación; potencial de aprendizaje; estudio longitudinal; inteligencia, evaluación dinámica.

\section{Introducción}

Durante más de 100 años la determinación de sobredotación intelectual se ha realizado de manera casi exclusiva en base a la puntuación de C.I.. No obstante, desde hace más de una década se viene planteando la falta de exactitud de la evaluación realizada sólo con tests tradicionales de inteligencia para la identificación de las altas capacidades, y se señala la necesidad de reconceptualizar la sobredotación intelectual, de incluir otras variables en su consideración, y de plantear alternativas diferentes para su diagnóstico (Calero, GarcíaMartín y Gómez, 2007; González Román, 2008; Joseph y Ford, 2006; Pfeiffer, 2012). Otras habilidades tales como el potencial de aprendizaje, los factores motivacionales y afectivos, la creatividad, flexibilidad, metacognición, memoria de trabajo e implicación en la tarea, se van considerando como necesarias para determinar la excelencia de un niño (Calero, García-Martín, Jiménez, Kazén y Araque, 2007; Joseph y Ford, 2006; Kaur, 2004; Lidz y Macrine, 2001; Naglieri y Ford, 2005; Sternberg y Dadvison, 2005; Tzuriel, Bengio, y Kashy-Rosenbaum, 2011). Aceptar esta postura implica entender que el CI que los niños superdotados manifiestan es el resultado de diferentes procesos y/o variables $y$, por ello, que aunque pueda ser un índice con alta fiabilidad no debe ser considerado como único criterio para establecer la sobredotación; menos aún, si la evaluación es temprana.

* Dirección para correspondencia [Correspondence address]: $\mathrm{M}^{\mathrm{a}}$ Dolores Calero. Facultad de Psicología, Universidad de Granada. 18071 Granada (España).E-mail: mcalero@ugr.es
Title: Temporal stability of IQ and learning potential in gifted children: diagnostic implications.

Abstract: Traditionally the determination of the giftedness has performed with traditional intelligence tests. The main argument to do so has been the temporal stability of this measure. In recent years some authors defend a determination early giftedness in children, although others point out that toddlers evaluation produces a significant number of false positives due to the variability in the measurement of intelligence by different factors such as plasticity, stimulation, etc.., therefore proposed the use of complementary indices for giftedness diagnosis. This paper presents a longitudinal study of two years to 49 children aged between 5 and 9 years, initially identified as gifted, to check the stability of your IQ and other measures such as the learning potential and working memory. The results show how learning potential and working memory measures remain stable in time while the IQ of a group of younger children is not maintained during this period. These results indicate the usefulness of P.A. measures as a supplementary index in the determination of the giftedness on young children.

Key words: I.Q.; giftedness; learning potential; longitudinal study; intelligence; dynamic assessment.

A pesar de estas posturas, la medida del C.I. sigue siendo el criterio más comúnmente utilizado debido fundamentalmente a que, en general, se considera una medida estable de la inteligencia, como se manifiesta en las altas correlaciones test-retest que para diferentes muestras de niños -incluidos superdotados- presentan los tests más reputados (Spranger y Sabatino, 1995; Ramsden et al., 2011). Podemos señalar los resultados aportados sobre el WISC-IV: .70 y .74 para seguimientos de hasta 3 años (Oakaman y Wilson, 1988); o para el K-Bit (Kaufman y Kaufman, 1997): .86 a .95, dependiendo del subtest (Mervis, Kisler, John y Morris, 2012).

Sin embargo se han aportado un considerable número de evidencias que señalan que el C.I. no es un atributo inmutable, sino que este puede cambiar por diferentes factores y no solo debido a un problema de fiabilidad o error de medida (Samenoff, Seifer Baldwin y Baldwin, 1993). Así por ejemplo Ramsden y colaboradores (2011) señalan como existen variaciones en las puntuaciones de niños y jóvenes que podrían relacionarse con cambios en la estructura cerebral debidos a diferentes factores tales como la edad, la estimulación, el sexo, etc..., o sea relacionables con la plasticidad cerebral. Sea este u otro el motivo, el caso es que se señalan cierto número de factores que intervienen en la variabilidad de las puntuaciones obtenidas a lo largo del tiempo por los tests más reputados, tales como el WISC (Lohman y Korb, 2006) o K-Bit -aunque este se haya mostrado como el test breve que mejor predice la sobredotación intelectual- (Mervis et al., 2012; Newton, McIntosh, Dixon, Willians y Youman, 2008)

La cuestión de la estabilidad de puntuaciones tiene una importancia considerable pues precisamente en la estabilidad 
temporal del C.I. en niños se basa la utilidad y validez de la detección temprana de niños superdotados.

Existen numerosos argumentos a favor y en contra de la identificación temprana de la sobredotación (Heller, 2004; Pérez y González, 2007) detrás de cada uno de ellos hay una teoría que justifica con qué, cuándo y para qué, realizar ese diagnóstico. Si repasamos la trayectoria de la investigación sobre esta práctica podemos comprobar el importante número de estudios que se han dirigido a la determinación intelectual de niños preescolares (Burns y Yumnard, 1991; Cukierkon, Karnes, Manning, Houston y Besnoy, 2007; Gottfried, Gottfried, Bathurst y Guerin, 1994; Sandel, McCallister y Nash, 1993; Sankar-DeLeeuw, 1999; Scott y Delgado, 2005). El objetivo fundamental para la defensa de esta identificación temprana ha sido casi siempre el de conseguir el máximo desarrollo para cada individuo y evitar posibles problemas de ajuste académico; tendencia que se apoya en la idea de que la sobredotación es una necesidad educativa más, y como tal, requiere una atención educativa adaptada (Chamberlin, Buchanan y Vercimak, 2007; Pérez y Gónzalez, 2007).

La mayoría de los defensores destacan además la importancia de aplicar un programa educacional lo antes posible a fin de desarrollar todo el potencial del niño (Benito, 2009; Burnside, 2007), con el fin de atender mejor las necesidades que en el niño se van planteando, o como medio de evitar la pérdida de talento en sus etapas iniciales (Pérez y González, 2007). A veces la explicación está basada en teorías biológicas que mantienen la creencia de que lo que no se utiliza o no se estimula adecuadamente se pierde (Benito, 2004; Burnside, 2007; Clark y Zimmerman, 2001; Lobo, 2005) y por ello la mayoría de los autores citan las ventajas de la identificación de preescolares superdotados con el objetivo de incluirlos en programas específicos (Cukierkon et al., 2007; Eriksson, 2006; Hodge y Kemp, 2006). En España, no hay apenas tradición de programas específicos para superdotados, y aún así, cierto grupo de investigadores sostienen también esta idea (Benito, 2004; Benito y Moro, 1997; Pérez y González, 2007).

Los padres son los mayores defensores de esta identificación temprana mientras que entre los maestros existen discrepancias y en un porcentaje considerable piensa que el diagnóstico no debería hacerse antes de los 6 años (SankarDeLeeuw, 1999); todo ello a pesar de que no existe transparencia a la hora de señalar la evidencia de la efectividad de esta medida (Comerford, Reid, Brain y Wilson, 2004) y de haberse incluso constatado que la incorporación temprana de los niños a los programas de sobredotación no garantizan los supuestos beneficios a largo plazo (Freedman, 2005). Así los seguimientos a largo plazo realizados en USA o Alemania (Arnold y Subotnik, 1994; Perleth y Heller, 1994; Schaie, 2005) parecen poner de manifiesto que las ventajas educativas tienden a desaparecer en pocos años (White, 1992).

Otros autores cuestionan la identificación temprana, por diversos motivos; en primer lugar no se entiende su utilidad cuando, en un contexto (el español, por ejemplo) no existe-, desde las instituciones, una respuesta general a las necesidades educativas de estos niños (Zavala, 2004); esto es, los programas educativos específicos desarrollados en nuestro país son minoritarios y dependen de los centros educativos o de las asociaciones. Por otro lado parece interesante tener en cuenta otros aspectos, además de la excelencia intelectual, para decidir la evaluación de un niño (Calero et al., 2007; Heller y Perleth, 2010; Joseph y Ford, 2006; Kaur, 2004; Lidz y Macrine, 2001, Naglieri y Ford, 2005; Sternberg y Dadvison, 2005); argumentos mucho más importantes son los que se centran en los problemas metodológicos derivados de la identificación temprana (Heller, 2004) pues según como ésta se haga puede conllevar mayor cantidad de falsos positivos cuanto menores sean los niños. Arnold y Subotnik (1994) muestran como a mayor edad se da una mayor fiabilidad en la predicción de la sobredotación y como ésta aumenta cuando se recogen diferentes informes sobre las tareas en las que el niño parece prometer e interesarse.

Autores como Silverman (1986) ya señalaron que muchos especialistas consideraban que no era adecuado identificar a los niños superdotados en sus primeros años. Argumentaba que las puntuaciones son imprecisas y que a la larga los demás niños se pondrían a su nivel. Las altas puntuaciones tempranas son sospechosas de estar demasiado influidas por un buen nivel de preescolar o por padres que estimulan mucho a sus hijos.

Sobre esta cuestión, algunos estudios como el de Pérez y González (2007) han tratado de analizar las diferencias en puntuaciones CI en tres grupos de edad según la etapa en que estaban escolarizados: educación infantil, educación primaria y educación secundaria, comparando un total de 148 niños. Los alumnos fueron propuestos para su evaluación por los profesores de sus centros o por las propias familias y a todos se les aplicó la escala Wechsler, WISC-R en el caso de los alumnos mayores de 6 años y el WIPPSI para los menores de esta edad. El estudio concluye que aquellas subescalas que tienen un mayor peso cultural y que contienen más elementos del currículum académico (Información, Semejanzas, Vocabulario y Aritmética) son las que aportan diferencias más significativas entre las tres etapas educativas siempre a favor de los grupos de mayor edad. Esto hizo a los autores plantearse una doble cuestión: por un lado que este tipo de materiales para detectar la capacidad intelectual están muy influidos por elementos culturales (con lo cual no serán igualmente eficaces para la detección de colectivos marginales, de riesgo o de bajo nivel cultural) y por otra parte, se cuestionaron si lo que estaban midiendo era la inteligencia.

En un estudio muy similar Sweetland, Reina y Tatti (2006) analizan los perfiles cognitivos -comparando las puntuaciones verbales frente a las de razonamiento y las totalesde 161 estudiantes superdotados evaluados con el WISC-III. En su trabajo concluyen que existen diferencias significativas entre las puntuaciones verbales y las puntuaciones de razonamiento en favor de las primeras. Destacan que la carga cultural que pueden tener las puntuaciones CI (sobre todo 
en el área verbal) es más alta que lo indicado en el manual del test. De igual forma, explican que una de las razones por la que los alumnos superdotados pueden puntuar significativamente más bajo en tareas manipulativas puede ser el factor tiempo en algunas de las tareas.

Uno de los estudios más recientes realizado en España sobre comparación de medidas de inteligencia en diferentes rangos de edad es el realizado por Benito, Moro y Alonso (2009). En este estudio se analiza la validez del test WISCIV. La investigación se llevó a cabo con 84 niños de edades comprendidas entre los 6 y los 16 años; de ellos, 51 niños puntuaron por encima de 130 y estos fueron los considerados como superdotados. Según este estudio, el índice del WISC-IV que mejor predice la puntuación del S-B (StandfordBinet) en los alumnos no superdotados es el índice de Razonamiento Perceptivo, obteniendo una correlación muy elevada. Sin embargo, el índice del WISC-IV que mejor predice la puntuación del S-B en los alumnos superdotados es el índice de Comprensión Verbal. Por tanto, concluyen que el índice RV (razonamiento verbal) del WISC-IV (el índice con mayor carga cultural) es el mejor de los índices para predecir superdotación. Igualmente señalan que la comprensión verbal es el índice que tiene mejor capacidad predictiva del rendimiento académico.

En definitiva, como ya hemos señalado actualmente existe un notable acuerdo en la consideración de que una simple puntación C.I. no es suficiente para determinar la sobredotación de un niño (Brown et al., 2005; Calero, García-Martín, y Robles, 2011; Noel y Edmunds, 2007; Worrell, 2009) sobre todo cuando ésta depende de la validez predictiva del test y de las variables que pueden influir en el rendimiento de un niño en un test. Son ya numerosos los estudios que apuestan por métodos más amplios y alternativos para identificar a un sobredotado (Lidz y Macrine, 2001; Naglieri y Ford, 2005; Swanson, 2006) considerando que la información que apor$\tan$ los tests de inteligencia es sobre el aprendizaje adquirido por el niño/a hasta ese momento, sin aportar información de hasta dónde puede llegar (Dixon, Cross y Adams 2001; Joseph y Ford 2006; Renzulli, 1994; Scott y Delgado, 2005; Sternberg, 2005).

Algunos autores han considerado opciones novedosas, así Van Tassel-Baska y colaboradores (Van Tassel-Baska y Brown, 2007; VanTassel-Baska, y Little, 2011) trabajan con tareas no verbales (menos influidas por el enriquecimiento del ambiente) y con protocolos basados en la ejecución más que en el rendimiento puntual en un test. Otros autores sugieren que la perspectiva vygotskiana puede proveer compresión útil sobre la sobredotación y sobre la importancia y la influencia de la interacción social en el desarrollo infantil (Simonton, 2009) Algunos autores, siguiendo esa perspectiva, han utilizado técnicas de potencial de aprendizaje comprobando que los niños sobredotados han mostrado un aprendizaje más eficiente y una ZDP mayor y con más cambios (Calero et al., 2011; Simonton, 2009; Tzuriel et al., 2011) en este sentido podemos señalar los trabajos de Borland y Wright, (1994) que comprueban que la introducción de observación, medidas de portafolio y evaluación dinámica aumentan la fiabilidad de la identificación de niños superdotados en escuelas urbanas de educación infantil a las que asisten niños económicamente desventajados en la ciudad de N. York. También Sibaya, (1996) y Matthews, (2002) utilizan esta metodología evaluativa para conseguir que niños africanos (en el primer caso) y latinos (en el segundo) sean identificados como superdotados e incorporados a programas específicos para altas capacidades en Estados Unidos. Los resultados de estos estudios sugieren que mediante evaluación dinámica (denominación anglosajona habitual para las técnicas de potencial de aprendizaje) se pueden identificar alumnos potencialmente superdotados que no son identificados mediante medidas estáticas, y además identificar niños que, puntuando muy alto en medidas estáticas, pueden no tener un potencial mucho mayor que cualquier niño de su edad.

En esta dirección Calero y colaboradores (Calero et al., 2011), trataron de comprobar si el supuesto fundamental que sustentaba estos trabajos, esto es, que los niños superdotados tienen un mayor y más generalizado potencial de aprendizaje que los niños con una inteligencia normal y los resultados confirmaron esta hipótesis.

Siguiendo con esta línea de trabajo se propone una investigación dirigida a comprobar la estabilidad temporal (por un periodo de dos años) de la medida de C.I. y de las medidas de potencial de aprendizaje en niños inicialmente superdotados para intentar buscar una alternativa evaluativa que limite las posibles causas de error que pueden afectar a la fiabilidad en la identificación de niños superdotados.

Los objetivos que persigue esta investigación han sido:

1.- Comprobar la estabilidad del C.I. evaluados en niños de 5 a 9 años en un seguimiento de dos años. $\mathrm{La} \mathrm{H}^{\circ}$ de partida es que algunos niños no van a mantener sus puntuaciones iniciales del C.I.

2- Comprobar la estabilidad de 3 medidas de P.A. y de una de memoria de trabajo evaluadas en niños de 5 a 9 años en un seguimiento de dos años. $\mathrm{La}^{\circ}$ de partida es que estas puntuaciones permanecerán estables de una a otra evaluación

3.- Analizar qué medidas de las realizadas en el inicio: C.I. potencial de aprendizaje y/o memoria de trabajo predicen mejor su puntuación C.I. a los dos años.

\section{Método}

\section{Participantes}

La muestra ha estado compuesta por 49 niños de 5 a 9 años de edad, $(M=6.73 ; D T=1.27)$ de entre los cuales 19 son mujeres y 30 hombres. La muestra ha sido recogida en diferentes colegios tanto públicos como privados de la ciudad de Granada. Las puntuaciones CI de los participantes se mueven en una horquilla desde 134 a 159 ( $M=144.89$; $D T=6.28)$, no existiendo diferencias significativas en C.I re- 
lacionadas con el sexo $\left(t_{(48)}=.468 ; p=.642\right)$ ni con los grupos de edad $\left(C h i^{2}=12.170 ; p=.432\right)$.

Tabla 1. Distribución de participantes en función de la edad y la puntuación inicial de C.I.

\begin{tabular}{lllll}
\hline Rango edad/C.I. & $135-139$ & $140-144$ & $145-149$ & +150 \\
De 5 a 7 años & 7 & 10 & 9 & 6 \\
De 8 a 9 años & 2 & 4 & 5 & 6 \\
\hline
\end{tabular}

\section{Instrumentos}

Test Breve de Inteligencia de Kaufman (Kaufman y Kaufman, 1994).- El test breve de inteligencia K-BIT es un test de screening, de rápida aplicación. El K-BIT tiene como objetivo medir la inteligencia verbal y no verbal en niños, adolescentes y adultos.

Esta prueba, consta de dos subtests: vocabulario y matrices. Aporta puntuaciones típicas para cada uno de los subtests así como una Global de CI Compuesto. El manual señala unos coeficientes de fiabilidad que van desde .76 a .94 para el subtest de Vocabulario; desde .74 a .93 para el subtest de Matrices y de .82 a .96 para el CI Compuesto. Respecto de la validez de constructo, el CI compuesto del KBIT tuvo una correlación de .80 con el CI global del WISC$\mathrm{R}$, de .75 con el WAIS-R y de .60 con la suma de las puntuaciones de procesamiento mental del K-ABC y de .75 con la de los subtest de conocimientos. Los dos subtests del K-BIT miden los mismos constructos que el $\mathrm{K}-\mathrm{ABC}$ y las escalas de Wechsler lo que evidencian su validez.

Test de Posiciones (Calero y Navarro, 2003).- Es una versión del Test de Fijación de Posiciones propuesto originalmente por Rey para evaluar la capacidad de fijación mnésica, utilizado por Feuerstein, Rand y Hoffman (1979), para la medida del potencial de aprendizaje y adaptado por Calero y Navarro, (2003). En su procedimiento, el examinador muestra cinco cruces dibujadas sobre una cuadrícula de 25 cuadrados. Luego pide al sujeto que reproduzca las posiciones indicadas marcándolas en cuadrícula similar en blanco. Hay cinco láminas diferentes y el sujeto tiene 8 ensayos como máximo para reproducirlas correctamente. Cada ensayo fallido implica un nivel de ayuda mayor por parte del examinador. Así, después del primer ensayo, la ayuda del evaluador consiste en señalar la localización de las cruces; tras en segundo ensayo se le pide al sujeto que sea él quien las señale; tras el tercero se dan indicaciones de tipo numérico; después del cuarto ensayo se dan ayudas de tipo espacial; y a partir del quinto ensayo, se aplican conjuntamente los dos niveles de ayuda anteriores. El formato de esta prueba es de entrenamiento dentro del test: se obtiene una puntuación ganancia resultado de sumar las puntuaciones obtenidas en cada uno de los 5 ítems puntuados de 1 a 8 dependiendo del número de ensayos que el sujeto haya necesitado para reproducir el ítem de forma correcta.
Test del organizador. Test adaptado del LPAD (Feuerstein et al., 1979).- La prueba evalúa la capacidad del sujeto para utilizar una información dada, con el fin de reunir nueva información mediante procesos de elaboración inferencial. Evalúa también la adquisición de estrategias y su aplicación para deducir relaciones a través de la evaluación y análisis de una información verbal compleja.

Consta de tres fases: pretest, aprendizaje y postest. Las tareas consisten en sistemas lógicos cerrados. En cada ítem se presenta una serie de declaraciones o premisas. Cada premisa permite la extracción de parte de la información requerida para reducir la incertidumbre y especificar con total precisión el emplazamiento o localización de una serie de entidades (objetos, colores, personas) en un campo dado. De su aplicación se obtiene 3 puntuaciones: Puntuación pretest (número de ítems resueltos correctamente de la primera parte del test que consta de 10 ítems), Puntuación postest (número de ítems resueltos correctamente de la segunda parte del test que consta de 20 ítems). Puntuación ganancia: Diferencia entre puntuación post y pretest.

Test de Diseño de Patrones. Test adaptado del LPAD (Feuerstein et al., 1979).- Se trata de una tarea de estructuración perceptiva que requiere el análisis y síntesis de una serie de estímulos realizados mediante la superposición de plantillas de diferentes colores y formas. Consta de veinte estímulos en los que se debe confeccionar por representación, -y no mediante mera manipulación motórica-, un diseño idéntico al que figura en el modelo coloreado. Los patrones coloreados, seis de los cuales son compactos y doce huecos, están impresos en un cartel gris, y el sujeto debe reproducir el diseño haciendo referencia a los patrones que deben utilizarse y especificando la secuencia en que deben superponerse entre sí. La forma, el color y la estructura del diseño específico determinan la selección de los patrones y el orden de su colocación. El formato es de entrenamiento dentro del test. De este se derivan 2 puntuaciones: Puntuación pretest (número de ítems resueltos correctamente sin ninguna ayuda). Puntuación de ganancia (puntuación resultado de la solución correcta sin ayudas del resto de los ítems, según los siguientes criterios: 4 puntos si logra resolver el ítem sin ayuda; 3 puntos si logra resolverlo con 1 ayuda; 2 puntos si lo resuelve con 2 ayudas y 1 si tenemos que ofrecerle 3 ayudas (número máximo de ayudas establecidas).

Esto tres tests fueron validados inicialmente por Feuerstein et al. (1979) para niños y posteriormente han sido utilizados por diferentes autores que han comprobado su utilidad y validez de constructo (para un conocimiento más extenso de estas pruebas remitimos a Feuerstein et al., 1979) (Calero, 2004; Calero y Navarro, 2003).

Tarea experimental de memoria de trabajo.- Para medir la memoria de trabajo hemos utilizado una tarea experimental de series de números (Oakhill, Yull y Parkin, 1989). En esta se suministran unas cartulinas con series de números que a su vez son leídas por el examinador. Se comienza con dos se- 
ries de números y se pide recordar el último número de cada serie. Hay hasta cinco series de números. La puntuación tiene un rango que va desde 0 a 5 .

\section{Procedimiento}

Todos los niños del estudio se evaluaron a través de un convenio de colaboración entre la Facultad de Psicología y la Asociación de niños Superdotados de Granada cuyo objetivo era la realización de informes de seguimiento de los niños de la asociación. Previo consentimiento informado de los padres se seleccionaron a los posibles participantes en este estudio. Los criterios de selección fueron la edad, de 5 a 9 años, y el diagnóstico previo de sobredotación. Todos los niños estaban identificados como tales por los equipos de orientación educativa y presentaban un C.I. superior a 130 determinado por el WISC o WIPPSI, según su edad. Estos niños pasaron una evaluación individualizada de unas 2 horas aproximadamente, en la que se le aplicó el K-bit y las diferentes tests del P.A. y memoria de trabajo, y transcurridos 2 años, se volvieron a evaluar con las mismas pruebas. De los 52 seleccionados inicialmente, tres no realizaron el seguimiento a los dos años.

\section{Diseño y Análisis Estadísticos}

El diseño seguido en esta investigación ha sido longitudinal con dos factores: el momento (evaluación inicial (1) y a los dos años (2)) y el grupo (niños superdotados que mantienen sus puntuaciones CI transcurridos dos años y niños superdotados que no mantienen sus puntuaciones CI en la evaluación a los dos años). Así el grupo mantienen estaba formado por aquellos niños $(n=27)$ cuya diferencia CI2 CI1 era positiva, 0 o negativa hasta tres puntos (error de medida del K-bit), - puntuación media de cambio del C.I. = -0.11; DT $=1.65$ - ; el grupo no mantienen $(n=22)$ estaba formado por aquellos niños cuya diferencia CI2 - CI1 era superior a -3 puntos, - puntuación media de cambio en $\mathrm{CI}=$ - 23.95; DT $=6.49$-.

Las variables independientes han sido las puntuaciones de C.I. total, C.I. verbal y C.I. matrices, puntuaciones en la tarea de memoria de trabajo y puntuaciones de ganancia simple en los tres tests de potencial de aprendizaje.

Los análisis estadísticos han sido: Modelo Lineal General de medidas repetidas con un factor de agrupación (mantienen / no mantienen) para analizar el comportamiento de las variables de inteligencia, memoria de trabajo y potencial de aprendizaje en su evaluación inicial y final, tanto a nivel intersujetos como intrasujetos, análisis discriminante para comprobar la eficacia predictiva de las medidas de inteligencia y las de potencial de aprendizaje sobre la puntuación C.I. total a los dos años, análisis de regresión entre estas mismas medidas y Chi cuadrado.

\section{Resultados}

Los resultados aportados por los estadísticos descriptivos nos informan que de entre los 49 niños y niñas que conforman la muestra de nuestro estudio un total de 22 niños, no mantienen sus puntuaciones CI en la evaluación realizada a los 2 años y 27 de ellos sí mantienen su nivel de CI. El primer grupo estaba formado por 13 hombres y 9 mujeres con una edad media de $5.86(D T=.35)$ mientras que el segundo estaba formado por 17 hombres y 10 mujeres con edad media de $7.44(D T=1.31)$. Existen por tanto diferencias significativas en edad entre ambos grupos $(t=6.008 ; p<.001)$.

Para comprobar las $\mathrm{H}^{\circ} 1$ y 2 que establecía el mantenimiento diferencial intergrupos (mantienen/ no mantiene) de las puntuaciones de C.I., de potencial de aprendizaje y de memoria de trabajo en los dos años transcurridos, se realizó un análisis según el Modelo Lineal General de medidas repetidas; este nos muestra, como puede observarse en la Tabla 2, que existen diferencias significativas entregrupos en todas las variables evaluadas, con tamaños de efecto moderados o altos. Así para C.I. total $(F(1 / 47)=148.484 ; p<.0001)$, C.I. ver$\mathrm{bal}(F(1 / 47)=39.820 ; p<.0001)$, C.I. matrices $(F(1 / 47)=$ $136.391 ; p<.0001)$, memoria de trabajo $(F(1 / 47)=209.709$; $p<.0001)$ ganancia en test de posiciones $(F(1 / 47)=$ $725.324 ; p<.0001)$, ganancia en test organizador $(F(1 / 47)$ $=90.205 ; p<.0001)$ y ganancia en diseño de patrones $(F$ $(1 / 47)=249.448 ; p<.0001)$. También la interacción entre las medidas repetidas (evaluación inicial $v$ s. a los dos años) y grupo (mantienen/no mantienen) es significativa para las tres medidas de inteligencia (C.I. total $(F(1 / 47)=338.246$; $p$ $<.0001)$, C.I. verbal $(F(1 / 47)=75.376 ; p<.0001)$ y C.I. matrices $(F(1 / 47)=106.988 ; p<.0001)$ con alto tamaño de efecto y potencia $=1$, mientras que esta interacción no se da en el resto de las variables evaluadas: potencial de aprendizaje en los tres tests utilizados y memoria de trabajo. Las Figuras 1 y 2 ejemplifican claramente estos resultados. 


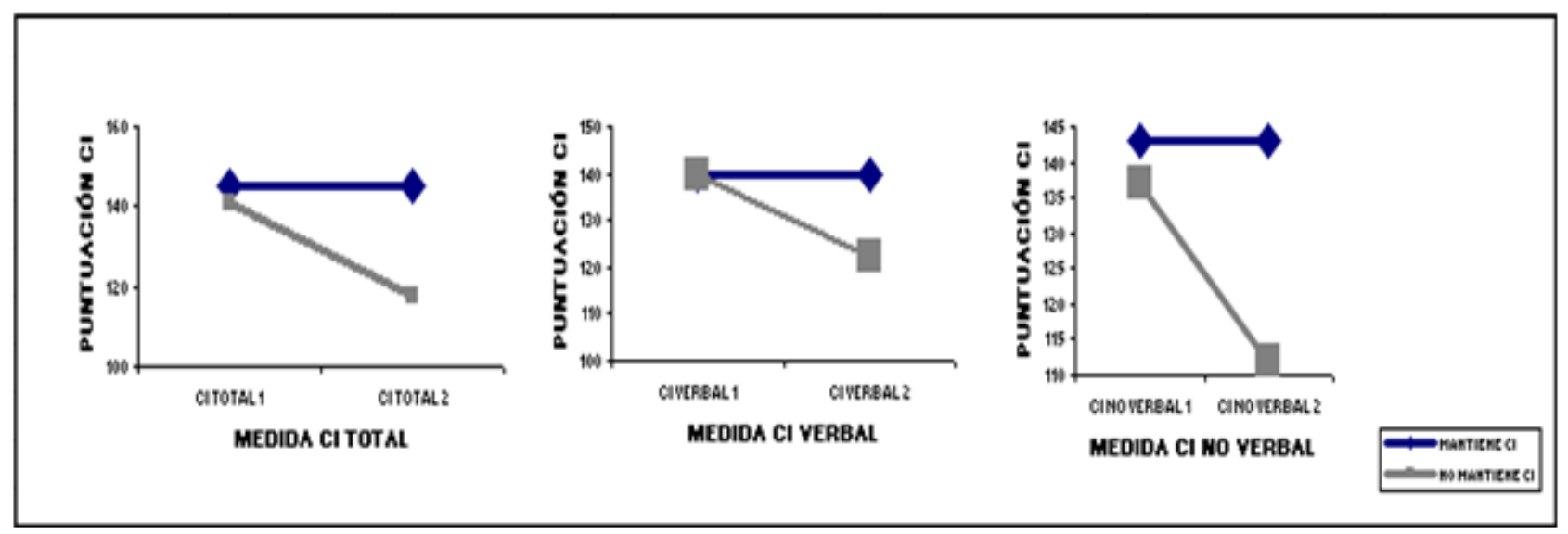

Figura 1. Medias inicial (1) y a los dos años (2) en las tres puntuaciones de inteligencia obtenidas del K-Bit para los dos grupos (mantiene CI vs no mantiene CI) de participantes.

Tabla 2. Diferencia de medias entre grupos (mantienen/ no mantienen) en la evaluación inicial (1) y la evaluación a los dos años (2).

\begin{tabular}{|c|c|c|c|c|c|c|c|c|c|c|c|c|}
\hline & \multicolumn{4}{|c|}{ MANTIENEN } & \multicolumn{4}{|c|}{ NO MANTIENEN } & \multicolumn{2}{|c|}{ INTERACCIÓN } & \multicolumn{2}{|c|}{ INTERGRUPOS } \\
\hline & \multicolumn{12}{|c|}{ EVALUACION 1 EVALUACION 2 EVALUACION 1 EVALUACION 2} \\
\hline & $\bar{M}$ & $D T$ & $D T$ & $M$ & $D T$ & $M$ & $D T$ & $M$ & $F_{(1 / 47)}$ & eta & $F_{(1 / 47)}$ & eta \\
\hline$\overline{\text { CI TOTAL }}$ & 147.37 & 5.60 & 147.26 & 5.29 & 141.86 & 5.80 & 117.91 & 5.14 & $338 . .246^{* *}$ & .878 & $148.484^{* *}$ & .760 \\
\hline CI VERBAL & 143.07 & 6.23 & 143.59 & 6.35 & 140.31 & 10.32 & 122.04 & 7.74 & $75.376^{* *}$ & .616 & $39.820 * *$ & .459 \\
\hline CI MATRICES & 143.85 & 5.70 & 143.81 & 5.07 & 136.68 & 11.03 & 112.36 & 5.39 & $106.988^{* *}$ & .695 & $136.391 * *$ & .774 \\
\hline M. DE TRABAJO & 4.58 & .37 & 4.71 & .33 & 3.22 & .35 & 3.27 & .38 & .958 & .020 & $209.709 * *$ & .817 \\
\hline POSICIONES & 38.88 & 1.25 & 39.85 & .45 & 31.45 & 1.40 & 31.77 & 1.84 & 2.520 & .037 & $725.324 * *$ & .939 \\
\hline GANANCIA & & & & & & & & & & & & \\
\hline $\begin{array}{l}\text { ORGANIZADOR } \\
\text { GANANCIA }\end{array}$ & 9.51 & .93 & 10.0741 & .95 & 6.72 & 1.60 & 6.27 & 1.98 & 5.310 & .108 & $90.205^{* *}$ & .657 \\
\hline $\begin{array}{l}\text { DISEÑOS } \\
\text { GANANCIA }\end{array}$ & 33.29 & 4.15 & 33.22 & 4.53 & 15.50 & 3.08 & 17.04 & 3.51 & 5.994 & .113 & $249.448^{* *}$ & .800 \\
\hline
\end{tabular}

** $p<.0001 ; * p<.05$

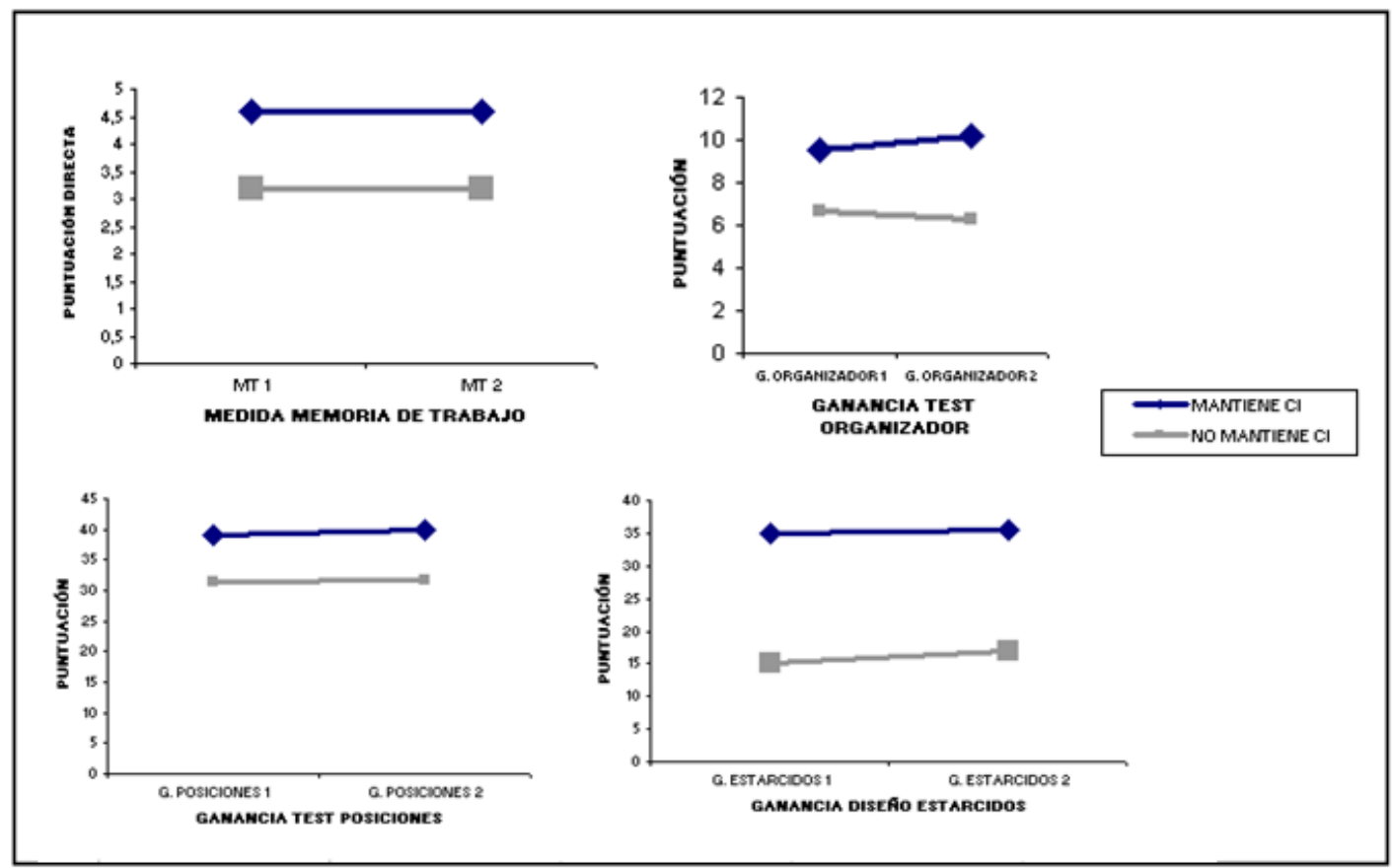

Figura 2. Medias de memoria de trabajo y de las tres medidas de potencial de aprendizaje en la evaluación inicial (1) y a los dos años (2) de los dos grupos (mantiene CI vs no mantiene CI) de participantes. 
La Tabla 3 nos muestra los resultados del análisis discriminante realizado para extraer las variables que mejor predicen el mantenimiento del CI a los dos años. De las medidas incluidas en el análisis: C.I. total, verbal y matrices, memoria de trabajo y medidas de ganancias de las tres pruebas, todas ellas recogidas en la evaluación inicial; las variables que aparecen como predictoras; son, por este orden, las puntuaciones de ganancia del Test de Posiciones (Paso 1: Lambda de Wilks = 0.109), y del Test del Organizador $(\mathrm{Pa}-$ so 2: Lambda de Wilks $=0.074$ ) así como la medida de memoria de trabajo (Paso 3: Lambda de Wilks $=.066 ; \chi^{2}=$ 123.57; $p$.0001), explicando el 100\% de la varianza. Las dos medidas de potencial de aprendizaje presentan también una alta correlación con el C.I. total a los dos años, en el análisis de regresión por pasos realizado: puntuación de ganancia en test de posiciones $\left(R^{2}=.791 ; F_{(1 / 148)}=177.475 ; p\right.$
$<.0001)$, la anterior más la puntuación de ganancia en el organizador $\left(R^{2}=.855 ; F_{(1 / 148)}=177.475 ; p<.0001\right) ; \mathrm{La}$ Tabla 4 presenta la clasificación que mediante esas medidas se hace el grupo muestral. Los resultados ponen de manifiesto cómo las pruebas con más peso predictor clasifican correctamente al $100 \%$ de los niños de la muestra de este estudio $\left(\chi^{2}(2 / 47)=45.041 ; p<.0001\right)$.

La Tabla 5 presenta la distribución entre el rango de edad y la pertenencia a los dos grupos (mantienen/ no mantienen). Como puede observarse, de los 49 niños, los 22 niños y niñas que no mantienen su CI pertenecen todos al grupo de niños de edades comprendidas entre los 5 y 7 años mientras que del grupo de niños de entre 8 y 9 años, no hay ninguno en que disminuya su puntuación CI en la segunda evaluación.

Tabla 3. Análisis Discriminante. Variables que superan en el análisis.

C.E.F.D. canónica: coeficientes estandarizados de la función discriminante canónica.

\begin{tabular}{|c|c|c|c|c|c|c|c|}
\hline PASO & VARIABLES & $F_{(1 / 47)}$ & $\begin{array}{l}L A M B D A \\
D E W I L K S\end{array}$ & $p$ & Autovalor & C. E.F.D. canónica & Correlación canónica \\
\hline 1 & POSICIONES GANANCIA & 383.458 & .109 & .0001 & 14.119 & & .966 \\
\hline \multirow[t]{2}{*}{2} & POSICIONES GANANCIA & 234.338 & .074 & .0001 & & & \\
\hline & ORGANIZADOR GANANCIA & 22.102 & & & & & \\
\hline \multirow[t]{3}{*}{3} & POSICIONES GANANCIA & 81.442 & .066 & .0001 & & .885 & \\
\hline & ORGANIZADOR GANANCIA & 13.477 & & & & .530 & \\
\hline & MEMORIA DE TRABAJO & 5.177 & & & & .339 & \\
\hline
\end{tabular}

Tabla 4. Tabla de contingencia de la distribución del grupo del estudio vs. el grupo pronosticado para el análisis.

\begin{tabular}{|c|c|c|c|}
\hline \multirow[t]{2}{*}{ GRUPO DEL ESTUDIO } & \multicolumn{2}{|c|}{ Grupo pronosticado para el análisis 1} & \multirow[t]{2}{*}{ TOTAL } \\
\hline & NO MANTIENEN CI & MANTIENEN CI & \\
\hline NO MANTIENEN CI MANTIENEN CI & 22 & 0 & 22 \\
\hline & 0 & 27 & 27 \\
\hline
\end{tabular}

$\underline{\chi^{2}(2 / 47)}=45.041 ; p<.0001$

Clasificados correctamente el 100,0\% de los casos agrupados originales.

Tabla 5. Tabla de Contingencia de la distribución entre grupos de edad y mantenimiento del C.I. a los dos años

\begin{tabular}{|c|c|c|c|}
\hline & NO MANTIENE & MANTIENE & TOTAL \\
\hline NIÑOS PEQUEÑOS (5- 7 AÑOS) & 22 & 10 & 32 \\
\hline NIÑOS MAYORES (8-9 AÑOS) & 0 & 17 & 17 \\
\hline TOTAL & $\chi^{2}(2 / 49): 19.358$ & $p<.0001$ & 49 \\
\hline
\end{tabular}

\section{Discusión y Conclusiones}

El objetivo general de este trabajo era comprobar la estabilidad de la medida de C.I. y del potencial de aprendizaje en un periodo de dos años en un grupo de niños inicialmente superdotados.

En primer lugar hemos de señalar que de los 49 niños evaluados, 22 disminuyen sus puntuaciones en CI en la evaluación que se hace a los dos años. Este hecho nos parece muy significativo y confirma muchas de las críticas que se han realizado respecto del uso de las técnicas de CI como único criterio para establecer la excelencia intelectual sobre todo cuando la evaluación se hace en edades tempranas (Arnold y Subotnik, 1996; Heller, 2004; Silverman, 1986).
En segundo lugar, cuando analizamos las diferencias iniciales entre el grupo de niños que mantienen su C.I. y los que no lo mantienen después de dos años, podemos observar cómo, aunque la media de ambos grupos se mantiene en el rango de la sobredotación intelectual, aparecen ligeras diferencias en el C. I inicial de esos grupos, a favor del grupo mantienen, en el C.I. total y C.I. matrices y las demás medidas de potencial de aprendizaje y memoria de trabajo, diferencias que son menores en el C.I. verbal que en el no verbal, estos resultados vuelven a confirmar los obtenidos por investigaciones anteriores sobre la influencia de los factores verbales en la estimación de la sobredotación (Benito et al. , 2009; Pérez y González, 2007; Sweetland et al. , 2006) .

Respecto a los dos primeros objetivos de este trabajo con los que tratábamos de comprobar las hipótesis sobre la 
estabilidad del CI y de las medidas de potencial de aprendizaje por un periodo de dos años, hemos podido comprobar cómo no aparecen diferencias significativas en las medidas de potencial de aprendizaje, ni de memoria de trabajo, de la primera evaluación a la segunda para el conjunto de los sujetos evaluados mientras que si se dan diferencias en las tres medidas de C.I.; esto es, el C.I. total, el verbal y matrices disminuyen significativamente de la primera a la segunda evaluación en un grupo numeroso de participantes. Estos resultados coinciden con lo señalado por algunos autores respecto de la estabilidad del C.I. mostrando como éste puede cambiar en el tiempo por diferentes factores tanto ambientales como biológicos o cognitivos (Lander, 2010; Ramsden et al. 2011; Samenoff et al.1993). Debemos considerar que las altas medidas test-retest que aportan los tests de inteligencia más reputados son por cortos periodos mientras que el tiempo transcurrido entre estas 2 evaluaciones ha sido de dos años, por lo que cabe pensar que más que una cuestión de fiabilidad del test se deba a un cambio cognitivo debido al desarrollo de estos niños. Sin embargo las medidas de potencial de aprendizaje, que se suponen evalúan la posibilidad de aprendizaje o modificabilidad cognitiva de los niños, permanecen estables en ese periodo de tiempo.

Es importante constatar como existe una diferencia significativa en la edad de los sujetos que componen ambos grupos, siendo el grupo de niños que no mantienen, los niños con edades comprendidas entre los 5 y 7 años.

Hace ya bastantes años, Silverman (1986) pensaba que no era adecuado identificar a los niños superdotados en sus primeros años ya que las puntuaciones podrían ser imprecisas y sospechosas de estar demasiado influidas por un buen nivel de preescolar o por la estimulación de los padres, otros autores apuntan a la plasticidad cerebral que poseen los niños en estos años como posible causa de su variabilidad individual en desarrollo intelectual (Ramsden et al, 2011).

Es importante resaltar así mismo como los niños que mantiene su estatus de superdotados han sido aquellos que, presentando inicialmente similares puntuaciones de inteligencia, destacaron por tener mayores puntuaciones de ganancia en los tests de potencial de aprendizaje y mayor memoria de trabajo. Como puede observarse en la tabla 5, no

\section{Referencias}

Arnold, K. D, y Subotnik, R. F. (1994). Lessons from contemporary longitudinal studies. En R. F. Subotnik y K. D. Arnold (Eds.), Beyond Terman: Contemporary longitudinal studies of giftedness and talent (pp. 2-51). Norwood, NJ: Ablex.

Benito, Y. (2004). Ventajas de la detección temprana del niño con talento y superdotado. Educar, 1, 25-34.

Benito, Y. (2009). Superdotación Intelectual. Conceptualización, identificación temprana. Pautas de identificación para padres, pediatras y profesores. Recuperado de http://www.cprceuta.es/Asesorias/ApoyoEducativo/ponencias $\% 20$ inc lusivi-

dad/Semana2/Superdotacion/Conceptualizaci $\%$ C3\%B3n, $\% 20$ Yolanda $\% 20$ Benito.pdf todos los niños pequeños disminuyen su puntuación C.I. sino que hay un $35.3 \%$ que lo mantienen, estos niños también presentaron alto potencial de aprendizaje inicial y este se ha mantenido en la evaluación realizada a los dos años.

Los resultados pues apuntan a la necesidad de utilizar pruebas complementarias al C.I. a la hora de establecer la sobredotación intelectual sobre todo cuando los niños son pequeños. También reflejan como los elementos verbales son los que pueden aparecer como más altos y dar lugar a falsos positivos en niños muy estimulados culturalmente (Silverman; 1986; Sweetland et al.,, 2006).

Por último, tratábamos de comprobar la capacidad predictiva sobre la estabilidad de las puntuaciones CI, de las distintas variables evaluadas y otra vez, dos medidas de P.A. y la medida de memoria de trabajo aparecen como predictores significativos, de tal modo que la clasificación mediante estos criterios consigue un acierto en la clasificación del $100 \%$ de los participantes. Estos resultados vienen a apoyar el amplio número de estudios que mantienen que las medidas de potencial de aprendizaje son más eficaces a la hora de identificar niños con sobredotación intelectual que las pruebas de inteligencia tradicionales incluso en niños cuya ejecución actual no sea sobresaliente, debido a que su evaluación no se dirige a estimar la ejecución conseguida sino el potencial de cambio positivo del evaluado (Calero et al., 2011; Noel y Edmunds, 2007; Renzulli, Gubbins, Del Siegle, Zhang, y Chen, 2005; Tzuriel et al. 2011).

En definitiva, dos resultados pueden ser destacados de este trabajo: en primer lugar la necesidad, para evitar falsos positivos sobre todo cuando se evalúa a niños pequeños, de utilizar diferentes tipos de registros y no solo pruebas de ejecución para determinar su nivel intelectual; en segundo lugar la utilidad de las medidas de potencial de aprendizaje para hacer un diagnostico de sobredotación más preciso, en línea con lo argumentado por Lidz y Macrine (2001). Debemos tener en cuenta, no obstante como limitaciones de este trabajo que reducen la generalizabilidad de las afirmaciones realizadas, que la muestra es reducida y que la prueba utilizada para medir C.I., aunque con alta validez diagnóstica, es breve. Creemos que la utilización de pruebas más extensas y mayor número de sujetos podrá dar luz sobre los resultados aquí obtenidos.
Benito, Y. y Moro, J. (1997). Proyecto para la Identificación Temprana de Alumnos Superdotados. Madrid, Ministerio de Educación y Cultura.

Benito, Y., Moro, J. y Alonso, J. A. (2009). ¿Qué es la inteligencia? Validez del test WISC-IV para medir la misma. Criterios de corrección para los alumnos con superdotación intelectual. Ideacción, 29, 2-53.

Borland, J. H. y Wright, L. (1994). Identifying young, potentially gifted, economically disadvantaged students. Gifted Chile Quarterly, 38(4) 164-171.

Burns, J. M. y Yunnard, J. D. (1991). Public programming for precocious preschoolers. Gifted Child Today, 6, 56-60.

Burnside, L. H. (2007). Psychological guidance of gifted children. Journal of Consulting Psychology, 6(4) 223-228. 
Brown, S. W., Renzulli, J. S., Gubbins, E. J., Del Siegle, W. Z. y Chen, C. H. (2005). Assumptions underlying the identification of gifted and talented students. The Gifted Child Quarterly, 49(1), 68-79.

Calero, M. D., García-Martín, M. B. y Gómez-Gómez, T. (2007). El alumno con Sobredotación Intelectual. Conceptualización, Evaluación y Respuesta Educativa. Sevilla, Consejería de Educación Junta de Andalucía.

Calero, M. D., García-Martín, M. B., Jiménez, M. I., Kazén, M. y AraqueCuenca, A. (2007). Self-Regulation advantage for High-IQ children: Findings from a research study. Learning and Individual Differences, 17(4) 328-343.

Calero, M. D., García-Martín, M. B. y Robles, M. A. (2011). Learning potential in high IQ children: The contribution of Dynamic Assessment to the identification of gifted children. Learning and Individual Differences, 21, 176-181.

Calero, M. D. y Navarro, E. (2003). El test de aprendizaje de posiciones: un instrumento de medida de la plasticidad del anciano deterioro cognitivo leve. Revista de Neurología, 36(7), 619-624.

Chamberlin, S. A. Buchanan, M. y Vercimak, D. (2007). Serving TwiceExceptional preschoolers: Blending gifted education and early childhood special education practices in assessment and program planning. Journal for the Education of the Gifted, 30(3), 372-394.

Clark, G. y Zimmerman, E. (2001). Identifying artistically talented students in four rural communities in the United States. Gifted Child Quarterly, 45(2), 104-114.

Comerford, L, Reid, I., Brain, K y Wilson, J. (2004) Accelerate learning: A literature survey. Unpublished report, Department of Education and Skills, London

Cukierkon, J. R., Karnes, F. A., Manning, S. J., Houston, H. y Besnoy, K. (2007). Serving the Preschool gifted child: Programming and resources. Roeper Review, 29(4), 271-276.

Dixon, F. A., Cross, T. L. y Adams, CH. M. (2001). Psychological characteristics of academically gifted students in a residential setting: a cluster analysis. Psychology in the Schools. 38(5), 433-45.

Eriksson, G. (2006). Applying multicultural a global education principles to the education of diverse gifted and talented children. En B. Wallace y G. Eriksson (Eds.), Diversity in gifted education: International perspectives on global issues. (pp. 1-9). New York: Taylor \& Francis Group.

Feuerstein, R., Rand, Y. y Hoffman, M. B. (1979). The Dynamic Assessment of Retarder Performers: The Learning Potential Assessment Device: Theory, Instrument and Techniques. Baltimore: U.P.P.

Freeman, J. (2005). Permission to be gifted: How conceptions of giftedness can change lives. En R. Sterberg y J.E. Dadvison (Eds.), Conceptions of giftedness (2 ed.) (pp. 80-97) N. York: Cambridge U. P.

Gónzalez Román, M. P. (2008). Análisis de la teoría PASS como modelo explicativo de la sobredotación. Faisca, 13(15), 77-92.

Gottfried, A.W., Gottfried, A. E., Bathurst, K. y Guerin, D. W. (1994). Gifted IQ early developmental aspects: The Fullerton longitudinal study. New York: Plenum.

Heller, K. A. (2004). Identification of giftedness and talented students. Psychology Science, 46, 302-323.

Heller, K. A. y Perleth, C. (2010) The Munich High Ability Test Battery (MHBT): A multidimensional, multhimethod approach. Faisca, 15(17), 18-35

Hodge, K. A. y Kemp, C. R. (2006). Recognition of Giftedness in the early years of school: Perspectives of teachers, parents and children. Journal for the Educational of the Gifted, 30(2), 164-282.

Joseph, L. M. y Ford, D. Y. (2006). Non-discriminatory Assessment: Considerations for Gifted Education. The Gifted Child Quarterly, 50(1), 42-53.

Kaufman, A. S. y Kaufman, N. L. (1997). Test Breve de inteligencia de Kaufman. Madrid: TEA.

Kaur, I. (2004). Adaptation of gifted students to the system of education in Malaysian secondary schools. Roeper Review, 26(3), 175-176.

Lander, J. (2010) Long-Term stability of scores on the Wechsler Intelligence Scale for Children $-4^{\circ}$ in children with learning disabilities. Dissertation Theses Farleigh Dickinson University (unpublished).

Lidz, C. S. y Macrine, S. L. (2001). An alternative approach to the identification of gifted culturally and linguistically diverse learners. School Psychology International, 22, 74-96.
Lohman, D. F., y Korb, K. A. (2006). Gifted today but not tomorrow? Longitudinal changes in ability and achievement during elementary school. Journal for the Education of the Gifted, 29, 451-484.

Lobo, M. P. M. (2005). A study of talent in students from early childhood and primary education. Electronic Journal of Research in Educational Psychology, 3(3), 109-120.

Matthews, M.S. (2002). Dynamic Assessment of academic ability of bilingual immigrant Latino children (Doctoral Theses no publicada). Universidad de Georgia: Disponible en red: hdl.handle.net/10724/5845.

Mervis, C. B., Kisler, D. J., John, A. E. y Morris, C. A. (2012) Longitudinal assessment of intellectual abilities of children with Williams Syndrome: Multilevel modeling of performance on Kaufman Brief Intelligence Test. Second edition. American Journal on Intellectual and Developmental Disabilities, 117 (2), 134-155.

Naglieri, J. A. y Ford, D. Y. (2005). Increasing Minority Children's Participation in Gifted Classes Using the NNAY: A Response to Lohman. The Gifted Child Quarterly, 49(1), 29-36.

Noel, K. y Edmunds, A. L. (2007). Constructing a Synthetic-Analytic Framework for precocious writing. Roeper Review, 29(2), 125-132.

Newton, J. H., McIntosh, D. E., Dixon, F., Willians, T. y Youman, E. (2008). Assessing giftedness in children: comparing the accuracy of three shortened measures of intelligence to the Stanford-Binet Intelligence Scales, Fifth edition. Psychology in the Schools, 45(6),523-536.

Oakhill, J., Yuill, N. y Parkin, A. (1989). Working Memory, comprehension ability and the resolution of text anomaly. British Journal of Psychology, 80, 351-361.

Oakaman, S. y Wilson, B. (1988). Stability for the WISC-R Intelligence Scores: implications or 3-year reevaluations for learning disabled students. Psychology in the School, 25(2), 118-120.

Pérez, L. y González, C. (2007). Diferencias evolutivas y variabilidad intelectual entre estudiantes con alta capacidad. Faisca, 12(14), 106-117.

Perleth, C. y Heller, K. A. (1994). The Munich longitudinal study of giftedness. En R. F. Subotnik y K. D. Arnold (Eds.), Beyond Terman: Contemporary longitudinal studies of giftedness and talent (pp. 77-114) Norwood, N.J.: Ablex

Pfeiffer, S. I. (2012). Current perspectives on the identification and assessment of gifted student. Journal of Psychoeducational Assessment, 30(1), 1-9

Ramsden, S. Richardson, F. M., Josse, G., Thomas, M. S. C., Ellis, C. Shakeshaft, C., Seghier, M. L. y Price, C.J. (2011) Verbal and non-verbal intelligence changes in the teenage brain, Nature, 479 , 113- 116. Doi: 10.1038 /nature10514.

Renzulli, J. S. (1994). El concepto de los tres anillos de la superdotación: un modelo de desarrollo para una productividad creativa. En Y. Benito (Coord.), Intervención e investigación psicoeducativas en alumnos superdotado (pp. 41-78). Salamanca: Amarú Ediciones,

Renzulli, J. S., Gubbins, E. J., Del Siegle, J., Zhang, W. y Chen, C. (2005). Assumptions underlying the identification of gifted and talented students. Gifted Child Ouarterly, 49(1), 68-79.

Samenoff, A. J. Seifer, R. Baldwin, A y Baldwin, C. (1993). Stability of intelligence from preschool to adolescence: the influence of social and family risk factors. Child Development, 64(1), 80-97

Sandel, A., McCallister, C. y Nash, W. R. (1993). Child search and screening activities for preschool gifted children. Roeper Review, 16, 98-102.

Sankar-Deleeuw, N. (1999). Gifted preschoolers: Parent and Teacher views on identification, early admission and programming. Roeper Review, 21(3), 174-179.

Schaie, K. W. (2005) Developmental influences on adult intelligence: The Seattle longitudinal study. N. York, Oxford U.P.

Scott, M. S. y Delgado, C. F. (2005). Indentifying cognitively gifted minority students in preschool. The Gifted Child Quarterly, 49(3), 199-213.

Sibaya, P. (1996). Giftedness and Intelligence Assessment in a third world country: Constraints and alternatives. Gifted Education International, 11(29), 107-113.

Simonton D. K. (2009). The "Other IQ": Historiometric assessment of intelligence and related construct. Review of General Psychology, 13(4), 315326

Silverman, L. K. (1986). Parenting young children. En J. R. Whitmore (Ed.), Intellectual giftedness in young children: Recognition and development (pp. 73-87). New York: Haworth. 
Spranger, R. S. y Sabatino, D. A. (1995). Temporal stability of Gifted children`s intelligence. Roeper Review, 17(3), 207-210

Sternberg, R. J. (2005). WISC: A Model of Giftedness in Leadership. Roeper Review, 28(1), 37-45.

Sternberg, R. J. y Dadvison, J. E. (Eds.) (2005) Conceptions of giftedness,(2 ed.),N. York: Cambridge I.P.

Swanson, J. D. (2006). Breaking through assumptions about Low-Income, minority gifted students. The Gifted Child Quarterly, 50(1), 11-27.

Sweetland, J. D., Reina, J. M. y Tatti, A. F. (2006). WISC-III Ver$\mathrm{bal} /$ Performance discrepancies among a sample of gifted children. The Gifted Child Quarterly, 50(1), 7-12.

Tzuriel, D. Bengio, E. y Kashy-Rosenbaum, G. (2011) Cognitive Modifiability, Emotional-motivational factors and behavioural characteristics among gifted versus non-gifted children. Journal of Cognitive Education and Psychology, 10(3), 253-269.
VanTassel-Baska, J., y Brown, E. F. (2007). Toward best practice: An analysis of the efficacy of curriculum models in gifted education. Gifted Child Quarterly, 51, 342-358.

VanTassel-Baska, J., y Little, C. (Eds.). (2011). Content-based curriculum for high ability learners (2nd Ed.). Waco, TX: Prufrock Press.

White, K. R. (1992). The relation between socioeconomic status and academic achievement. Psychological Bulletin, 91, 461-481.

Worrell, F. C. (2009). A Myth 4: single test score or indicator tells use II: we need to know about giftedness. The Gifted Child Quartelerly, 53(4), 242244

Zavala, M. A. (2004). Desarrollo y validación de un sistema para la detección de alumnos con aptitudes sobresalientes-superdotados. Revista de Educación y Desarrollo, 14-20.

(Artículo recibido: 2-12-2012; revisado: 17-4-2013; aceptado: 10-7-2013) 